\title{
ORIGINAL ARTICLE PREVALENCE OF SILENT CARDIAC ISCHEMIA IN TYPE II DIABETES MELLITUS
}

\author{
Nosheen Zia ${ }^{1}$, Sabeen Aftab ${ }^{1}$, Nauman Ismat Butt ${ }^{1}$, Fahmina Ashfaq ${ }^{1}$, Aniqa Anser ${ }^{1}$, \\ Samina Saeed ${ }^{2}$ \\ ${ }^{1}$ Chaudhary Muhammad Akram Teaching and Research Hospital, Azra Naheed Medical College Lahore, Pakistan, Jinnah Hospital, \\ ${ }^{2}$ Allama Iqbal Medical College Lahore, Pakistan
}

\begin{abstract}
Objectives: To determine the frequency of silent cardiac ischemia in type II diabetes mellitus patients presenting to tertiary care facility.

Methodology: This cross-sectional study was conducted from $20^{\text {th }}$ October 2017 to $20^{\text {th }}$ April 2018 at Department of Medicine Jinnah Hospital Lahore. After a detailed history, measurement of height, blood pressure, weight, fasting blood sugar, body mass index of 237 patients were recorded, and electrocardiography (ECG) was done before and after exercise tolerance testing (ETT). Changes in ECG were assessed and person labeled as having silent cardiac ischemia or not depending on the outcome of the ECG.

Results: Mean age was $54.9 \pm 5.9$ (range: $45-63$ ) years. There were 144 male patients and 93 female patients The mean duration of type II diabetes mellitus in the study population was 10.0 \pm 2.6 (range: $6-15$ ) years. The frequency of silent cardiac ischemia was found to be $45.1 \%$. Increasing age, prolonged duration of diabetes mellitus, history of smoking and hypertension, and increased body mass index were significantly related to silent $\mathrm{cardiac}$ ischemia ( $\mathrm{p}$-value $<$ $0.05)$.
\end{abstract}

Conclusion: Silent cardiac is quite prevalent in our population that demands urgent efforts to diagnose this condition earlier for better future outcomes.

Keywords: Silent cardiac ischemia, type II diabetes mellitus, BMI, hypertension

Citation: Zia N, Aftab S, Butt NI, Ashfaq F, Anser A, Saeed S. Prevalence of Silent Cardiac Ischemia in Type II Diabetes Mellitus. Pak Heart J. 2021;54(02):162-166. DOI: https://doi.org/10.47144/phj.v54i2.2093

\section{INTRODUCTION}

Cardiovascular complication is an important cause of mortality in type II diabetes, accounting for $65 \%$ to $85 \%$ deaths. ${ }^{1}$ Coronary heart disease is often more marked in diabetics and it also may have an atypical presentation. Silent cardiac ischemia (SCI) is an objectively documented cardiac ischemia while lacking features of chest discomfort or other anginal equivalents. ${ }^{2}$ In the US, silent ischemia may be evident in approximately up to 3 million patients with stable coronary artery disease (CAD). ${ }^{1}$ During their lifetime almost $40 \%$ of diabetic patients with heart disease develop short-term episodes of cardiac ischemia; and $75 \%$ do not develop symptoms, hence labeled 'silent'. ${ }^{3}$ The most commonly used clinical diagnostic tools for cardiac ischemia in routine practice are ETT and ambulatory ECG, or Holter monitoring: ${ }^{4}$ Exercise tolerance testing (ETT) is the preferable test to detect silent ischemia in patients with either asymptomatic or symptomatic disease. However diagnosis of CAD in asymptomatic patients should be confirmed by radionuclide imaging (thallium perfusion scintigraphy or exercise ventriculography). The US Preventive Services Task Force (USPSTF), the American Heart Association, and the American College of Cardiology have not suggested ETT use as a screening tool due to insufficient data. ${ }^{5}$ However, the American Diabetes Association recognizes the plausible efficacy of ETT in diabetics with multiple CAD risk factors, for patients with high-risk occupations at risk of CAD, for men $>45$ years and women $>55$ years who wish to initiate a vigorous exercise program. ${ }^{6}$ For confirmation or rebuttal of the presence of SCI in patients having a positive exercise test result ambulatory monitoring is recommended which assesses ECG changes while the subject engages in daily routine activities. ${ }^{7}$

Silent cardiac ischemia is usually a misunderstood clinical entity. The cause of silent ischemia is often attributed to autonomic neuropathy complicating diabetes mellitus. ${ }^{8}$ It has been hypothesized that a combination of parasympathetic and sympathetic pathway abnormalities may be responsible. Studies have shown that diabetic patients having silent exertional ischemia, had evidence of remarkable autonomic impairment in spite of lacking overt microvascular complications, indicating that subclinical neuropathy plays a significant role in causing silent ischemia. With respect to risk of acute coronary events or sudden cardiac death, silent cardiac ischemia is not different from symptomatic coronary artery disease. As a predictor of severe coronary heart disease, 40 to $90 \%$ patients 
with SCI show findings of coronary artery stenosis on coronary angiography. ${ }^{9} \mathrm{SCI}$ is usually estimated by measuring ischemic ECG changes during and soon after an exercise tolerance/ stress test in otherwise healthy asymptomatic diabetic patients.

Literature regarding the frequency of silent cardiac ischemia in Asian patients with type II diabetes is scarce. The estimated frequency of silent cardiac ischemia in type II diabetic patients has been reported to fluctuate between $20 \%$ to $50 \% .^{10}$ In retrospect however some authors did not observe any significant difference in the frequency of silent ischemia in diabetic patients when compared to non-diabetic subjects. Since we do not have convincing data from Pakistani population regarding frequency of cardiac ischemia in diabetic patients, therefore, this study was designed to determine the frequency of silent cardiac ischemia in patients with type II diabetes mellitus and considered sound and rationalized. It would help us in creating awareness on the topic in light of its results later on to reduce disease mortality and morbidity and to strategize screening to improve quality of life.

\section{METHODOLOGY}

This cross-sectional study was conducted at Medical Department, Jinnah Hospital Lahore from $20^{\text {th }}$ October 2017 to $20^{\text {th }}$ April. After approval from Ethical Review Board of Jinnah Hospital, 237 patients aged 20 to 60 years belonging to either gender with type II diabetes mellitus (DM) for $\geq 5$ years and physically fit for exercise tolerance test having normal resting (baseline) ECG with no previous history of coronary heart disease were included in the study. Patients with history of acute coronary syndromes (unstable angina, NSTEMI, STEMI), hear failure, CVA, history of uncontrolled cardiac arrhythmia or AV block, current pregnancy, Liver disease (AST, ALT > $40 \mathrm{IU} / \mathrm{L}$ ) and patients on digitalis therapy, or using beta-blockers were excluded from the study. The sample size of 237 was calculated with an absolute precision of $5 \%$ and 95\% confidence level considering anticipated population proportion of $19.0 \%$ for silent cardiac ischaemia. ${ }^{11}$ After taking a written formal informed consent, demographic information including patient's height, weight, pulse, blood pressure, and respiratory rate were noted.

Laboratory investigations for haemoglobin and fasting lipid profile were done from Pathology Laboratory of Allama Iqbal Medical College. A baseline twelve-lead resting ECG was performed in all subjects and analyzed for any abnormal changes. Each patient underwent a structured clinical assessment to document co-morbidities prior to exercise testing. Major coronary risk factors including history of obesity, hypertension, duration of diabetes mellitus, hyperlipidemia, family history of coronary heart disease and smoking history were documented. An exercise tolerance test was conducted on the subjects using Precor® treadmill to detect any ischemic changes in otherwise asymptomatic subjects with no prior evidence of coronary heart disease.

ECG tracings were taken at peak of exercise and immediately after the end of exercise. During recovery blood pressure and ECG were monitored at two minutes, five minutes and ten minutes after completion of the exercise tolerance test. Maximal ST-Segment change at 80 milliseconds after the Jpoint was analyzed on the ECG tracings. Horizontal or down sloping ST -segment depression $>1 \mathrm{~mm}$ or up sloping of $>1.5 \mathrm{~mm}$ were labeled as a positive test result for silent cardiac ischemia. The collected data was analyzed using SPSS version 20.0. The data were stratified according to age, gender, hypertension, blood sugar levels, BMI, smoking ( $>5$ packs per year) and their effect were seen on prevalence of silent cardiac ischemia by applying chi-square test. A p-value of $\leq 0.05$ was taken statistically significant.

\section{RESULTS}

Demographic characteristics are shown in Table 1. One hundred and seven $(45.1 \%)$ patients exhibited silent cardiac ischemia. Seventy two $(50 \%)$ male patients and $35(37.0 \%)$ female patients showed silent cardiac ischemia. Though the prevalence of silent cardiac ischemia was clinically seen more in male patients however, the effect was statistically not significant with p-value: 0.062 . Out of the 93 hypertensive patients, $71(76.4 \%)$ had silent cardiac ischemia. Out of a total of 144 patients who did not have hypertension, 36 (25.0\%) still exhibited silent cardiac ischemia. The difference in the frequency of silent myocardial ischemia in hypertensive and nonhypertensive patients was statistically significant with p-value: 0.0001 .

Out of 83 patients with positive history of smoking in our study, $71(85.5 \%)$ patients had silent cardiac ischemia. However, of the non-smoker group, 36 $(23.4 \%)$ out of a total of $154(100.0 \%)$ patients had evidence of silent cardiac ischemia. The difference between smokers and non-smokers was statistically significant with p-value: 0.00001. Age, duration of diabetes mellitus, fasting blood sugar level, body weight and body mass index were significantly related to the silent cardiac ischemia with $\mathrm{p}$-value: $0.0001, \quad 0.002,0.00001, \quad 0.00001$ and 0.01 respectively. Body Height was not significantly 
related to body the silent cardiac ischemia with $\mathrm{p}$ value: 0.145 .

Table 1: Demographic Characteristics of the patients $(\mathbf{n}=237)$

\begin{tabular}{|l|c|}
\hline Age & $\begin{array}{c}54.9 \pm 5.9 \text { years } \\
\text { (range: } 45-63)\end{array}$ \\
\hline Duration of Disease & $\begin{array}{c}10.0 \pm 2.6 \text { years } \\
\text { (range: } 6-15)\end{array}$ \\
\hline Height & $\begin{array}{c}70.5 \pm 11.2 \mathrm{~kg} \\
\text { (range: } 55-95)\end{array}$ \\
\hline Body mass index & $\begin{array}{c}66.0 \pm 4.2 \text { inches } \\
\text { (range: } 60.0-73.0)\end{array}$ \\
\hline Fasting blood sugar & $\begin{array}{c}25.9 \pm 2.4 \mathrm{~kg} / \mathrm{m}^{2} \\
\text { (range: } 23-32)\end{array}$ \\
\hline Sex & $\begin{array}{c}170 \pm 18 \mathrm{mg} / \mathrm{dl} \\
\text { (range: } 138-202)\end{array}$ \\
\hline Male & $144(60.8 \%)$ \\
\hline Female & $93(39.2 \%)$ \\
\hline Hypertension & $93(39.2 \%)$ \\
\hline Present & $114(60.8 \%)$ \\
\hline Absent & $83(35.0 \%)$ \\
\hline Smoking Status & $154(65.0 \%)$ \\
\hline Smoker & \\
\hline Non-smoker &
\end{tabular}

\section{DISCUSSION}

The notion that silent cardiac ischemia is linked to serious and life-threatening cardiac events is plausible biologically, given the remarkable parallels in the circadian occurrences of cardiac ischemia, MI and sudden death, along with their reduction by use of $\beta$ blockers. Histopathological studies also support the notion that recurrent ischemia might lead to irreversible cardiac changes linked to the formation of scarred or fibrotic myocardium, which acts as a catalyst for developing fatal arrhythmias, or subsequently congestive cardiac failure. ${ }^{12}$ However patient-based studies that support the development of arrhythmic disturbance or heart failure linked to the detection of ischemia are deficient. Even though silent ischemia (either on exercise testing or ambulatory monitoring) in the general population is associated with a higher relative risk of serious cardiac events, poor sensitivity and specificity of these tests make them less reliable screening tools. In particular Ambulatory ECG monitoring could not be used in mainstream practice for this capacity. In the stable convalescent period after MI or in stable angina, silent ischemia detected by ambulatory monitoring does not sum information from exercise testing and clinical assessment about the likelihood of re-infarction or death. Ischemia during stress testing or on exercise ECG does predict the likelihood of developing new hard cardiovascular events. This is vastly affected by functioning capacity and the threshold above which ischemia occurs, but less likely by the occurrence of concurrent pain during the test. This is different for the acute coronary syndromes, including STEMI, for which ongoing transient ischemia is linked with an increased risk of infarction and death irrespective of whether it is silent or not and the duration of ischemia is proportional to the increase in risk. Moreover, transient ischemia may help stratify patients who would aid from treatment with antithrombotic therapy, making continuous ECG monitoring a highly useful tool in this clinical scenario.

The knowledge of silent ischemia has enlightened our understanding of many pathophysiological events underlying the natural history of coronary disease. It has become apparent over the years that the detection of symptomatic or silent ischemia can be of considerable diagnostic and prognostic value when the clinical population under study is clearly defined. Moreover meticulous selection of the study population along with the detection techniques, are essential for the search for silent ischemia to reach its maximal clinical vitality. Depending on the population under study, the criteria used to define silent ischemia used and the mechanism of ischemia detection utilized the prevalence estimates of SCI vary by a large degree. In the cohort by Framingham study ${ }^{13}$ patients who had unremarkable ECG at baseline with no findings of CAD were followed till 30 years, using biennial clinical review and ECG. More than one in four of the cardiac infarctions identified were previously undiagnosed, and up to half of them remained silent. The identification of undetected ischemia does not essentially mean that the ischemia was silent but perhaps the ischemia was unrecognized because the patients had misinterpreted atypical angina or had not exercised adequately in their routine lives to develop symptoms of ischemia. Numerous studies appear to highlight the high frequency of silent ischemia in patients with diabetes but are limited due to the varying definitions of SCI used between studies. Nomura et al. ${ }^{14}$ conducted study of 925 patients with type II diabetes who undertook exercise stress test (EST) followed by exercise thallium scintigraphy if the EST was abnormal, showing that abnormal stress test was abnormal in $12.1 \%$ and an abnormal response in both tests for $6.4 \%$ patients. In the 2004 study, Detection of SCI in Asymptomatic Diabetic Subjects (DIAD) 22\% patients with type II diabetes with no detectable CAD had SCI using EST and adenosine technetium-99 single-photon emission-computed tomography cardiac perfusion imaging. ${ }^{15}$ Our study reveals much higher frequency of cardiac ischemia than these studies showing the high burden of the disease in our population which needs greater steps to combat its consequences in patient's lives.

The question, why type II diabetics have a greater prevalence of SCI as compared to the general population? has various expositions (compromising a variable threshold of pain perception and psychological denial) but autonomic neuropathy has a significant role, possibly causing functional disturbance at various levels including the pain receptors, the afferent neurons and the 
neurological mechanisms leading till supratentorial translation of ischemia into pain. The autopsy study ${ }^{16}$ of a diabetic patient whose death was caused by a silent cardiac infarction, identified pathologic changes in afferent cardiac neurons suggesting a neuropathy, and the extended anginal threshold detected in patients with type II diabetes has link with reduction of heart rate variability, an initial sign of cardiac autonomic nerve dysfunction (CAN). Furthermore, the inability to detect angina at the start of ischemia will have a lax effect on the exercise tolerance: the affected patients will exercise more in the absence of chest pain and are at risk to develop severe ischemia consequently. ${ }^{16}$ Patients with type II diabetes showed significantly less MIBG uptake compared to healthy controls, further supporting the suggestion that sympathetic denervation is directly linked to abnormalities in perception of cardiac pain in diabetic patients. ${ }^{17}$ The relevant occurrence of autonomic dysfunction in diabetic patients is also supported by the absence of a peak incidence of cardiac ischemia during the morning hours. ${ }^{12}$ Finally, diabetic patients with or without signs of autonomic neuropathy have a lower vagal activity (and hence a relatively greater sympathetic activity) during late night hours and this is the time when most of the cardiovascular events are most frequently reported. ${ }^{12}$

After adjustment for other known prognostic indicators, the occurrence of silent episodes of cardiac ischemia on ECG monitoring, remained an independent mortality predictor. ${ }^{18}$ The outcomes of SCI for the patients are manifold: the acute cardiac infarction would go unrecognized causing delay in the commencement of medicines proven to decrease mortality post-infarction, such as heparin and beta-blockers, additionally the patients will spend the immediate post-infarct period at their routine activities at risk of an post-infarct arrhythmia out-of-hospital rather than under cardiac monitoring. This leads to the fact that diabetics have more established coronary artery disease at first presentation compared to non-diabetics and higher risk of having multi-vessel disease.$^{19}$ Furthermore, the 5-year survival rate after their first major cardiac event for diabetic patients was 38\% and only $25 \%$ after subsequent events, as compared to the corresponding $75 \%$ and $50 \%$ respectively in nondiabetics. Recently, a significantly higher mortality rate with PTCA than with CABG in patients with diabetes mellitus and multivessel coronary disease, 5-year mortality data showed that patients taking insulin or oral therapy have a mortality rate of $35 \%$ with PTCA compared with $19 \%$ mortality rate with $\mathrm{CABG} .{ }^{20}$ Diabetic patients also have an increased risk of reinfarction and a higher need for additional revascularization procedures, possibly due to early restenosis. However, further studies should be performed assessing whether stent implantation will reduce the mortality rate in diabetic patients after multi-vessel PTCA.

The risk of cardiovascular disease in conjunction with the high risk of SCI in diabetics would strongly favor a screening strategy to detect silent coronary artery disease in diabetics to limit the possible consequences of undetected cardiac ischemia. However, screening remains a disputable idea. Firstly no single screening test shows adequate specificity to limit the requirement for invasive testing if the initial screening test is positive. Second, the profit of diagnosing asymptomatic coronary artery disease without an infraction remains ambigious. However, the American Diabetes Association (ADA) suggests diabetes to be considered as a coronary artery disease equivalent and aggressive treatment of the risk factors for coronary artery disease in diabetics irrespective of presence or absence coronary artery disease. ${ }^{21}$ Therefore, while diagnosing SCI in a patient with diabetes may result in measures such as attempted revascularization or commencement of beta-blockers, direct evidence is lacking that these measures improve prognosis in diabetics without a cardiac infarction and who are symptom free. In 2009, the DIAD group published the first large-scale prospective randomized controlled study about screening for SCI15 in which 1123 patients were followed for 5 years, showing that screening with MPI did not affect the likelihood of occurrence of a major cardiovascular event. The Bypass Angioplasty Revascularization Investigation (BARI) 2 Diabetes Trial failed to show any significant improvement in cardiovascular outcomes in patients treated with coronary revascularization therapy as compared to intensive medical therapy alone. ${ }^{22}$ Currently, the ADA recommends that screening for occult cardiac ischemia be performed only in patients with atypical or typical cardiac symptoms and an abnormal resting ECG representing a change in policy from the previous $\mathrm{ADA}$ recommendation to screen all patients with diabetes who have two or more additional risk factors for cardiovascular disease. ${ }^{22}$ Many studies have showed that the detection of silent ischemia during exercise testing or AECG monitoring depicts worse clinical outcome and poor survival. A threefold increase in cardiac deaths was reported in patients with silent ischemia during AECG monitoring at 2 year follow-up compared with those with no silent ischemia ( $24 \%$ versus $8 \%) .{ }^{13}$ Numerous other studies depict that despite control of anginal symptoms with anti-angina drugs, during daily routine activities more than $40 \%$ of patients with stable angina would continue to still have ECG evidence of cardiac ischemia on AECG monitoring. As most antianginal drugs are prescribed and titrated to control symptoms only, silent ischemia during routine life activities may remain undetected unless ECG is monitored. ECG, being an inexpensive test, has a high specificity to detect prior cardiac infarction when 
abnormal although not sensitive for lesser degrees of ischemia. $^{22}$ Administration of beta-blockers and revascularization have shown to benefit patients with a prior cardiac infarction; therefore, diagnosing a new ECG abnormality would lead to interventions that can improve prognosis. In conclusion, it is pertinent that patients with type II diabetes have higher morbidity and mortality from cardiovascular disease than non-diabetic patients; with diabetes being an independent strong risk factor for the occurrence of atherosclerosis, predisposing to the development of other known cardiac risk factors such as hypertension and hyperlipidemia.

\section{CONCLUSION}

Silent cardiac ischemia is a frequent occurrence in patients with type II diabetes, probably as a consequence of cardiac autonomic dysfunction. Age, history of smoking, history of hypertension, fasting blood sugar level, body weight and body mass index significantly affect the prevalence of silent cardiac ischemia in our study population. The presence of silent cardiac ischemia has important implications for the patient and physicians caring for such patients should look out to the possibility of silent ischemia.

\section{AUTHORS' CONTRIBUTION}

NZ: Concept and design, data acquisition, interpretation, drafting, final approval, and agree to be accountable for all aspects of the work. SA, NIB, FA, AA, SS: Data acquisition, interpretation, drafting, final approval and agree to be accountable for all aspects of the work.

Conflict of interest: Authors declared no conflict of interest.

\section{REFERENCES}

1. Kahn SE, Cooper ME, Del Prato S. Pathophysiology and treatment of type 2 diabetes: Perspectives on the past, present, and future. Lancet. 2014;383:1068-83.

2. Davis TME, Coleman R, Holman RR. Prognostic significance of silent myocardial infarction in newly-diagnosed type 2 diabetes: UKPDS 79. Circulation. 2013;127(9):980-7.

3. Malhotra S, Sharma R, Kliner DE, Follansbee WP, Soman P. Relationship between silent myocardial ischemia and coronary artery disease risk factors. J Nucl Cardiol. 2013;20(5):731-8.

4. Rozanski A, Gransar H, Hayes SW, Min J, Friedman JD, Thomson LEJ, et al. Temporal trends in the frequency of inducible myocardial ischemia during cardiac stress testing: 1991 to 2009 . J Am Coll Cardiol. 2013;61(10):1054-65.

5. American Diabetes Association. Standards of Medical Care in Diabetes--2014. Diabetes Care. 2014;37(Supp 1):S14-80.

6. Ferket BS, Genders TSS, Colkesen EB, Visser JJ, Spronk S, Steyerberg EW, et al. Systematic review of guidelines on imaging of asymptomatic coronary artery disease. J Am Coll Cardiol. 2011;57(15):1591-600.

\section{Address for Correspondence:}

Dr. Nauman Ismat Butt, Chaudhary Muhammad Akram Teaching and Research Hospital, Azra Naheed Medical College Lahore, Pakistan.

Email: nauman_ib@yahoo.com
7. Turakhia MP, Ullal AJ, Hoang DD, Than CT, Miller JD, Friday $\mathrm{KJ}$, et al. Feasibility of Extended Ambulatory Electrocardiogram Monitoring to Identify Silent Atrial Fibrillation in High- risk Patients: The Screening Study for Undiagnosed Atrial Fibrillation (STUDY- AF). Clin Cardiol. 2015;38(5):285-92.

8. Fisher VL, Tahrani AA. Cardiac autonomic neuropathy in patients with diabetes mellitus: current perspectives. Diabetes, Metab Syndr Obes. 2017;10:419-34.

9. Sultan A, Perriard F, Macioce V, Mariano- Goulart D, Boegner $\mathrm{C}$, Daures J, et al. Evolution of silent myocardial ischaemia prevalence and cardiovascular disease risk factor management in Type 2 diabetes over a 10- year period: an observational study. Diabet Med. 2017;34(9):1244-51.

10. Darouian N, Agrawal S, Wei J, Nelson MD, van Dam P, Laks M, et al. Prevalence Of Silent Myocardial Ischemia On Ambulatory Monitoring In Women With Coronary Microvascular Dysfunction. J Am Coll Cardiol. 2018;67(13 Supplement):2096.

11. Sheikh A, Faisal SS, Jabbar A. Frequency of silent myocardial ischaemia in diabetics: a single centre study. J Pak Med Assoc. 2011;61(10):1037-41.

12. Verrotti A, Prezioso G, Scattoni R, Chiarelli F. Autonomic neuropathy in diabetes mellitus. Vol. 5, Front in Endocrinol (Lausanne). 2014;205.

13. Mahmood SS, Levy D, Vasan RS, Wang TJ. The Framingham Heart Study and the epidemiology of cardiovascular disease: a historical perspective. Lancet. 2014;383(9921):999-1008.

14. Nomura T, Kusaba T, Kodama N, Terada K, Urakabe Y, Nishikawa S, et al. Clinical characteristics of silent myocardial ischemia diagnosed with adenosine stress $99 \mathrm{~m}$ Tc-tetrofosmin myocardial scintigraphy in Japanese patients with acute cerebral infarction. Heart Vessels. 2013;28(1):27-33.

15. McCarthy MM, Frans JT, Davey J, Chyun DA. Physical inactivity and cardiac events: An analysis of the Detection of Ischemia in 2017;9:8-14

16. Yu L, Liang H, Dong X, Zhao G, Jin Z, Zhai M, et al. Reduced silent information regulator 1 signaling exacerbates myocardial ischemia-reperfusion injury in type 2 diabetic rats and the protective effect of melatonin. J Pineal Res. 2015;59(3):376-90.

17. Everett BM, Brooks MM, Vlachos HEA, Chaitman BR, Frye RL, Bhatt DL. Troponin and cardiac events in stable ischemic heart disease and diabetes. N Engl J Med. 2015;373(7):610-20.

18. Bhatt DL, Bonaca MP, Bansilal S, Angiolillo DJ, Cohen M, Storey $\mathrm{RF}$, et al. Reduction in ischemic events with ticagrelor in diabetic patients with prior myocardial infarction in PEGASUS-TIMI 54. J Am Coll Cardiol. 2016;67(23):2732-40.

19. Farrer M, Reid PG, Albers CJ, Goodfellow J, Reid DS, Furniss SS, et al. 1017-109 Revascularisation and Quality of Life: Improved Self-Perceived Well-Being, Following PTCA or CABG Surgery. J Am Coll Cardiol. 2018;25(2 Supplement 1):392A-393A.

20. Varga Z V, Giricz Z, Liaudet L, Haskó G, Ferdinandy P, Pacher $P$. Interplay of oxidative, nitrosative/nitrative stress, inflammation, cell death and autophagy in diabetic cardiomyopathy. Biochim Biophys Acta (BBA)-Molecular Basis Dis. 2015;1852(2):232-42.

21. Ghasemzadeh N, Brooks MM, Vlachos H, Hardison R, Sikora S, Sperling L, et al. An Aggregate Biomarker Risk Score Predicts High Risk of Near- Term Myocardial Infarction and Death: Findings From BARI 2D (Bypass Angioplasty Revascularization Investigation 2 Diabetes). J Am Heart Assoc. 2017;6(7):e003587.

22. Leon BM, Maddox TM. Diabetes and cardiovascular disease: Epidemiology, biological mechanisms, treatment recommendations and future research. World $\mathrm{J}$ Diabetes. 2015;6(13):1246. Asymptomatic Diabetics (DIAD) study. J Clin Transl Endocrinol. 